\title{
Effect of tonic immobility induction on selected physiological parameters in Oryctolagus cuniculus f. Domesticus rabbits
}

\author{
ANNA WILCZYŃSKA ${ }^{1}$, JERZY ZIĘTEK ${ }^{1}$, OLIWIER TEODOROWSKI², \\ STANIS $Ł A W$ WINIARCZYK ${ }^{1}$, ŁUKASZ ADASZEK ${ }^{1}$
}

\author{
${ }^{1}$ Department of Epizootiology and Clinic of Infectious Diseases, Faculty of Veterinary Medicine, \\ University of Life Sciences in Lublin, Głęboka 30, 20-612 Lublin, Poland \\ 2"Teodorowscy" Veterinary Clinic, Żwirki i Wigury 5, 43-190 Mikołów, Poland
}

Wilczyńska A., Ziętek J., Teodorowski O., Winiarczyk S., Adaszek Ł.

Effect of tonic immobility induction on selected physiological parameters in Oryctolagus cuniculus $f$. Domesticus rabbits

\section{Summary}

Tonic immobility (TI) is a phenomenon known as thanatosis or apparent death. The phenomenon can be induced in many ways. It occurs, for example, in the presence of a hazard, as a result of coercion through an unnatural body position or as a result of administration of certain medicines. TI is sometimes used in veterinary practice to tame patients. The aim of the study was to determine the effect of tonic immobility induction on selected physiological parameters in Oryctolagus cuniculus $f$. Domesticus rabbits. The study included 20 healthy rabbits, patients of a veterinary clinic, divided into two groups of 10 individuals. In the study group, TI was induced to conduct a standard clinical trial, while the control group were subjected to a standard clinical trial without TI. Heart rate, respiratory rate, pupil diameter and blood glucose concentration were measured in all animals. The measurements were performed three times: after the patient's arrival at the clinic, after a detailed clinical examination in a normal or tonic immobility position and $\mathbf{1 5}$ minutes after the end of the examination. Additionally, the blood cortisol concentration was measured twice: on arrival at the clinic and at the end of the clinical trial. The results of the experiment show that miniature rabbits feel less stress during tonic immobility testing than rabbits in the standing position, and therefore this phenomenon should be used for taming animals.

Keywords: tonic immobility, rabbit, physiology

Tonic immobility (TI) is an innate behaviour characterized by temporary inhibition of motor functions $(20$, 22). It is also referred to as akinesis, thanatosis, apparent death and paralysis. This phenomenon is reversible and its duration is individually variable and genetically conditioned $(8,9)$. Studies carried out on various animal species (rats, domestic guinea pigs, rabbits) have shown that TI lasts from a few seconds to a few hours $(4,8,25)$. There are several symptoms characteristic of TI, such as stillness, unnatural body position, lack of reaction to external stimuli, tachycardia/bradycardia $(9,10)$, decrease in respiratory rate, decrease in body temperature (16) and changes in EEG.

The TI reaction occurs in vertebrates (e.g. rabbits, guinea pigs, mice, monkeys) and invertebrates (e.g. beetles, crustaceans, hymenoptera) $(3,7,14)$. In some species (e.g. chickens, turtles, sharks) it is used in research and diagnostic procedures $(2,23,24)$, and is induced by forcing a non-physiological position of the body: lying on the back $(5,6,15)$.

TI may be a defence response, and a similar mechanism is induced in some young mammals while being carried by their mothers ("mother's grip") $(5,6,15)$. In some species (cats, rabbits, ferrets), a kind of stillness occurs as an element of sexual behaviour $(5,6,15,21)$.

Studies carried out on rabbits so far have shown that animals in a state of severe stress, necessary for the induction of TI, give the impression of being calm (9, 19). Stressful situations activate the sympathetic nervous system, which is manifested by increased blood pressure and tachycardia, dilation of muscular blood vessels, constriction of the blood vessels in the digestive system, bronchial dilatation, pupil dilation, increased physical activity and renin production (1).

Many studies contain detailed descriptions of methods of inducing TI in rabbits, the most popular of which 
is to lay the rabbit on its back. Since breeders, rabbit owners and veterinarians believe that the TI procedure can be highly stressogenic for animals, the aim of this study was to determine how the induction of TI in rabbits by placing them in the dorsal position affects their physiological parameters indicating their level of stress.

\section{Material and methods}

The study was conducted from June 2018 to May 2019. It included 20 miniature rabbits (10 males and 10 females), patients of the Small Mammals Department of the Faculty of Veterinary Medicine in Lublin, weighing $600 \mathrm{~g}-2.5 \mathrm{~kg}$ (1.5 kg), aged 3-84 months (26 months). The animals were kept at home as companion animals. The rabbits were divided into two groups: control group ( 5 females and 5 males) and study group ( 5 females and 5 males). The control group of 10 rabbits were examined in a standing position. The study group of 10 rabbits were examined after being placed in the dorsal position to induce TI. Blood for biochemical and other tests was taken from the marginal vein of the ear as part of the clinical procedure. The study was conducted in accordance with the EU Convention on the protection of animals used for scientific purpose (Revised Directive 86/609/the EEC Directive of the European Parliament and of the Council on the protection of animals used for scientific purposes).

The animals were clinically examined, and their blood glucose and cortisol levels were determined. The first measurement set (Stage I) occurred after the owner entered the office and included the following parameters: respiratory rate, pulse rate, pupil size, and glucose and cortisol levels in the venous blood. The rabbits were then subjected to a detailed clinical examination, which lasted about 5 minutes, in the standing or dorsal position (tonic immobility). The second measurement set (Stage II) took place directly after the clini- cal trial, and included measurements of respiratory rate, pulse rate, pupil size and blood glucose concentration. This was followed by a 15 -minute interval prior to the third measurement set (Stage III), which included the determination of respiratory rate, heart rate, pupil size and the glucose and cortisol levels in the blood.

The pulse rate was measured with a MedAir PulseSense VET Pulse Oximeter. The sensor was placed on the median ear artery. The number of breaths was counted by observing respiratory movements of the chest. The pupil diameter was measured with a caliper.

Blood for biochemical tests was taken from the marginal vein of the ear. Blood serum cortisol concentration was determined by a Vcheck V200 analyzer using the VetExpert Vcheck Cortisol diagnostic kit. It is used for quantitative measurements of the concentration of cortisol in blood serum by the fluorescence method.

Blood glucose testing was performed with a Contour Plus One glucometer.

Statistical analysis. The results were compiled statistically by calculating the arithmetic mean and standard deviation for each parameter with the Statistica 13.1 EN program.

\section{Results and discussion}

Respiratory rate. In the control group, the respiratory rate during Stage I was $96-227 / \mathrm{min}$. In Stage II, this rose to $108-234 / \mathrm{min}$. At the end of the clinical trial, in Stage III, the rate was 108-228/min. (Fig. 1). During the period between Stages I and III, two animals showed a decrease in respiratory rate of $8 \%$, while the remaining animals showed an increase of $17 \%$ on average (Fig. 2). In the study group, the respiratory rate was $102-210 / \mathrm{min}$. in Stage I, 66-186/min. in Stage II, and $90-188 / \mathrm{min}$. in the final measurement (Stage III)

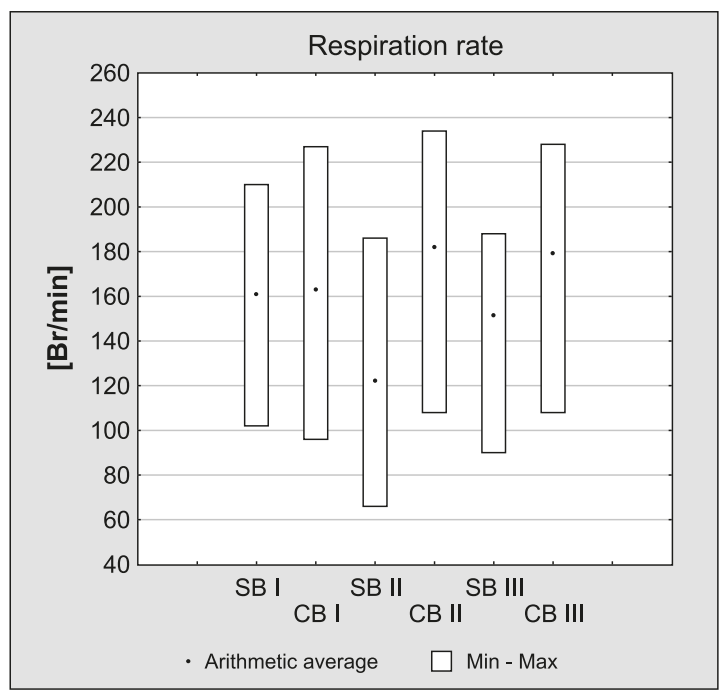

Fig. 1. Changes in respiratory rate in the study and control groups in subsequent measurements Explanations: SBI - respiratory rate in the study group in measurement 1 ; CBI - respiratory rate in the control group in measurement 1; SBII - respiratory rate in the study group in measurement 2; CBII - respiratory rate in the control group in measurement 2; SBIII - respiratory rate in the study group in measurement 3 ; CBIII - respiratory rate in the control group in measurement 3

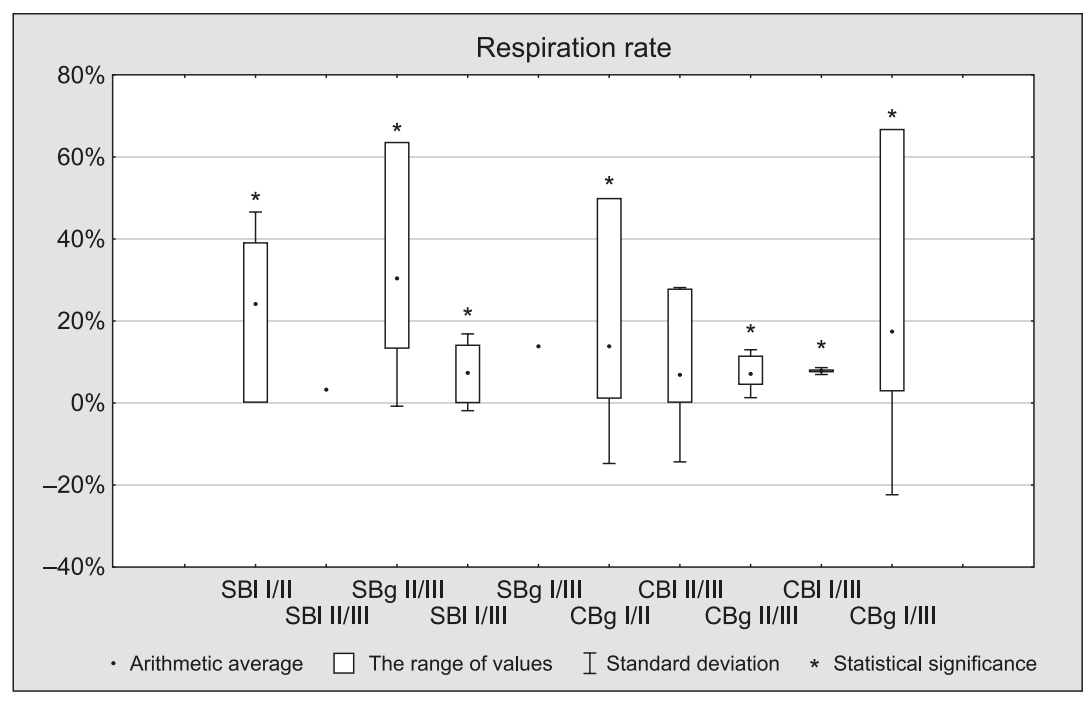

Fig. 2. Percentage change in respiratory rate between initial measurement and measurement during tonic immobility - I/II; between measurement during tonic immobility and final measurement - II/III; between initial measurement and final measurement - I/III

Explanations: $\mathrm{S}$ - study group; $\mathrm{C}$ - control group; 1 - decrease in value; $\mathrm{g}$ increase in value 


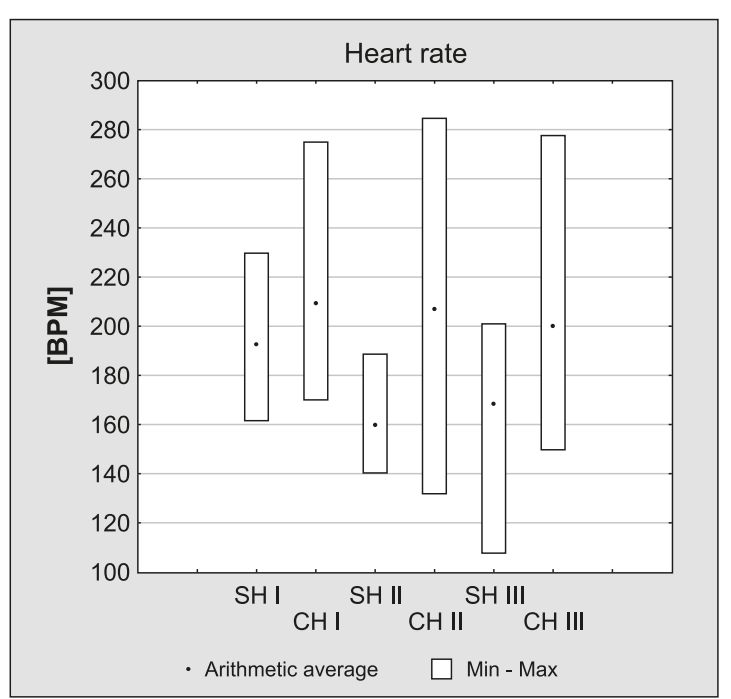

Fig. 3. Changes in heart rate in rabbits in the study and control groups in subsequent measurements

Explanations: $\mathrm{SHI}$ - heart rate in the study group in measurement 1; CHI - heart rate in the control group in measurement 1; SHII - heart rate in the study group in measurement 2; CHII - heart rate in the control group in measurement 2; SHIII - heart rate in the study group in measurement 3 ; CHIII - heart rate in the control group in measurement 3

(Fig. 1). Comparison of the measurements taken at the beginning and end of the observation shows that the respiratory rate decreased by $7 \%$ in 9 subjects and increased by 14\% in 1 animal (Fig. 2).

Heart rate. The heart rate in the control group was $170-275 / \mathrm{min}$ in Stage I, 132-285/min in Stage II, and $150-278 / \mathrm{min}$ in Stage III, at the end of the clinical trial (Fig. 3). At the end of the observation, 6 subjects had

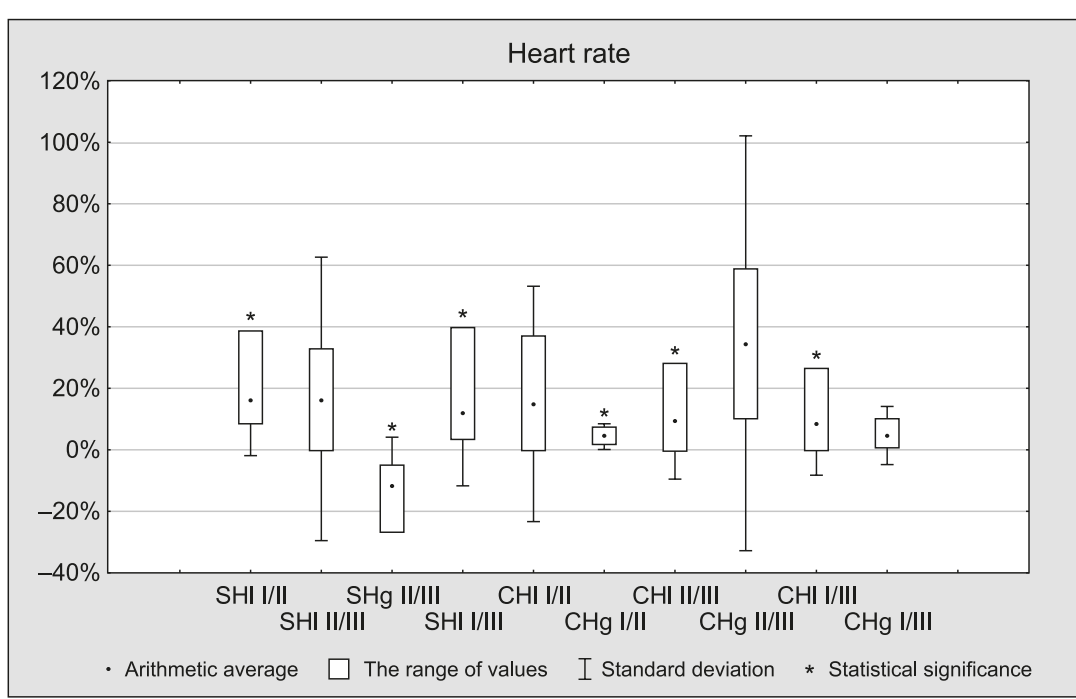

Fig. 4. Percentage change in heart rate between initial measurement and measurement during tonic immobility - I/II; between measurement during tonic immobility and final measurement - II/III; between initial measurement and final measurement - I/III

Explanations: $\mathrm{S}$ - study group; $\mathrm{C}$ - control group; 1 - decrease in value; $\mathrm{g}$ increase in value

an average decrease in the heart rate of $9 \%$, while 3 had an average increase of 5\% (Fig. 4). The heart rate in the study group was 162-230/min. in Stage I, 140-89/min during tonic immobility (Stage II) and 108-201/min at the end of the clinical trial (Stage III) (Fig. 3). Between the first and last measurements, the heart rate in all rabbits decreased on average by $12 \%$ (Fig. 4).

Pupil size. The pupil size in rabbits of the control group was 0.5-0.8 cm in Stage I, 0.6-0.9 $\mathrm{cm}$ in Stage II, and $0.4-0.9 \mathrm{~cm}$ in Stage III, at the end of the clinical trial (Fig. 5). In comparing pupil diameters at the beginning and end of the study (Stages 1 and 3), it was

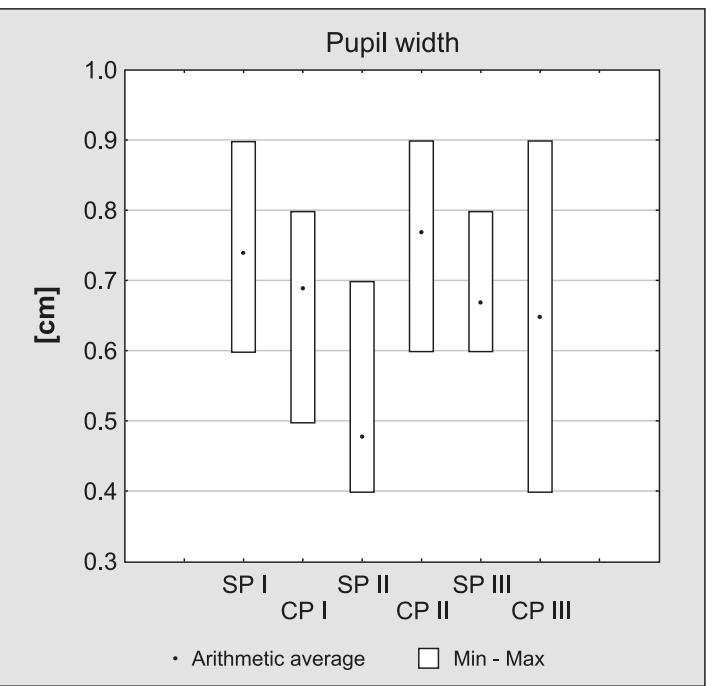

Fig. 5. Changes in pupil size in rabbits in the study and control groups in subsequent measurements Explanations: SPI - pupil size in the study group in measurement 1; CPI - pupil size in the control group in measurement 1; SPII - pupil size in the study group in measurement 2; CPII - pupil size in the control group in measurement 2; SPIII - pupil size in the study group in measurement 3; CPIII - pupil size in the control group in measurement 3

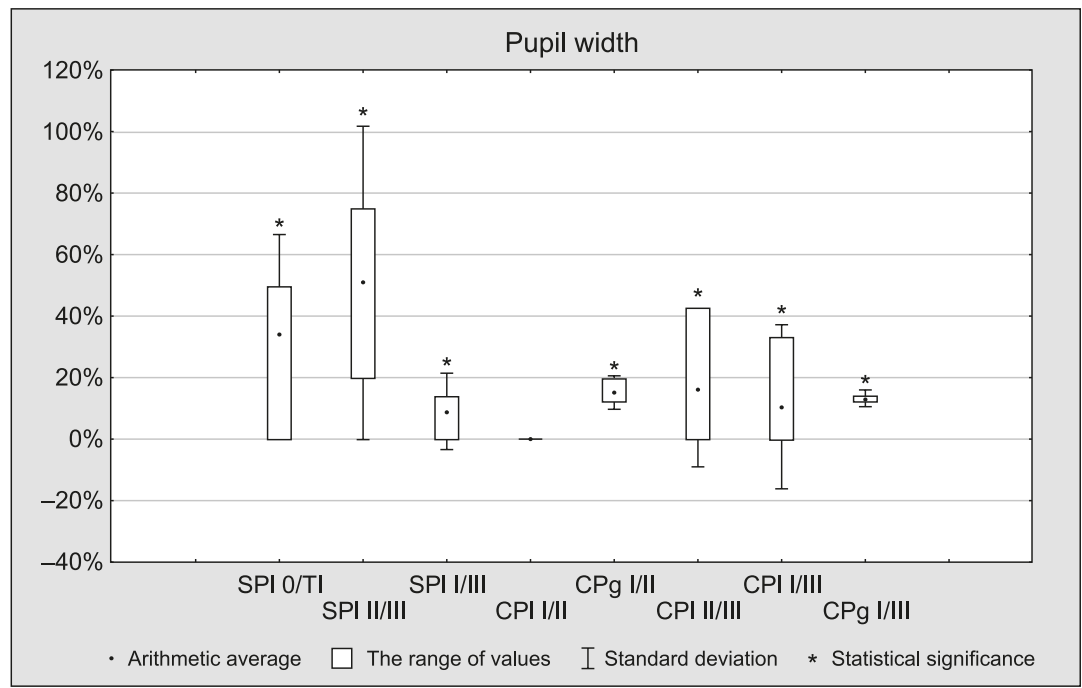

Fig. 6. Percentage change in pupil diameter between initial measurement and measurement during tonic immobility - I/II; between measurement during tonic immobility and final measurement - II/III; between initial measurement and final measurement - I/III

Explanations: $\mathrm{S}$ - study group; $\mathrm{C}$ - control group; 1 - decrease in value; $\mathrm{g}-$ increase in value 


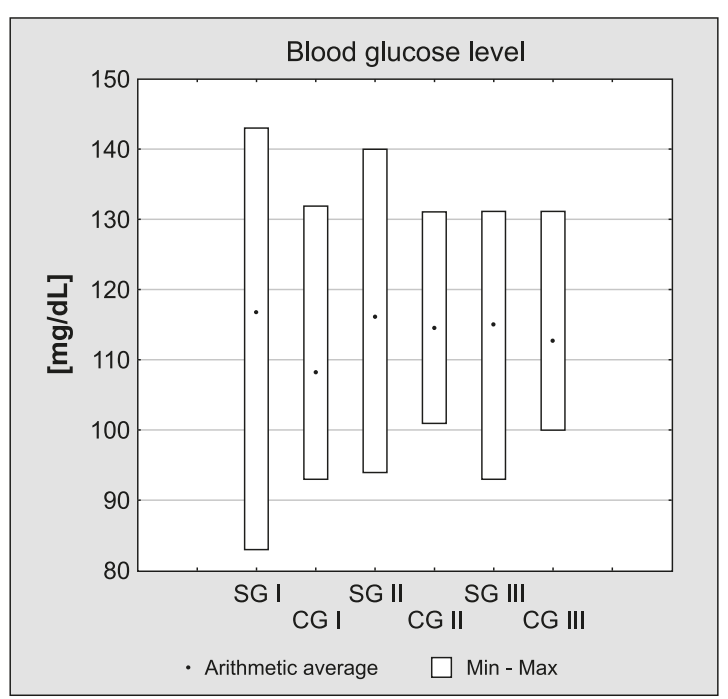

Fig. 7. Changes in glucose concentration in rabbits in the study and control groups in subsequent measurements

Explanations: SGI - glucose concentration in the study group in measurement 1; CGI - glucose concentration in the control group in measurement 1; SGII - glucose concentration in the study group in measurement 2; CGII - glucose concentration in the control group in measurement 2; SGIII - glucose concentration in the study group in measurement 3 ; CGIII - glucose concentration in the control group in measurement 3

shown that, on average, the diameter increased by $13 \%$ in 2 subjects and decreased by $11 \%$ in 7 (Fig. 6). The mean diameter of pupils in the study group was 0.6 $-0.9 \mathrm{~cm}$ after the introduction of animals into the study, 0.4-0.5 cm during the tonic immobility, and 0.6-0.8 at the end of the TI (Fig. 5). In comparing pupil diameters at the beginning and end of the study (Stages 1 and 3), it was shown that in all subjects of the study group the diameter decreased on average by $9 \%$ (Fig. 6 ).

Blood glucose concentration. The blood serum glucose concentration in rabbits from the control group was 93-132 $\mathrm{mg} / \mathrm{dl}$ (i.e. within the reference range) in Stage I, 101-131 mg/ dl during the clinical trial and 100-131 $\mathrm{mg} / \mathrm{dl}$ at the end of the trial (Fig. 7). Comparison of glucose concentrations at the beginning and end of the study (Stages 1 and 3 ) revealed that, on average, its level decreased by $3 \%$ in 3

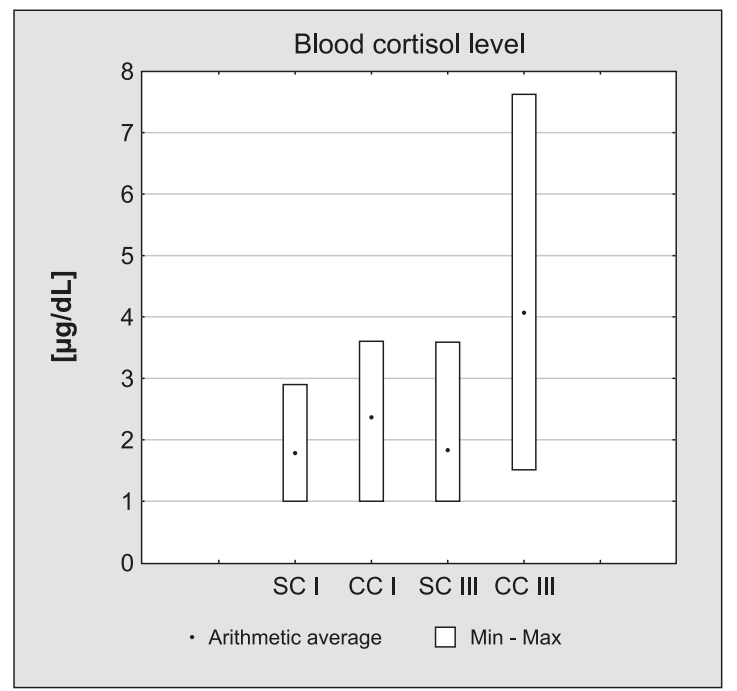

Fig. 9. Changes in cortisol concentration in rabbits in the study and control groups in subsequent measurements

Explanations: SCI - cortisol concentration in the study group in measurement 1 ; CCI - cortisol concentration in the control group in measurement 1 ; SCIII - cortisol concentration in the study group in measurement 3; CCIII - cortisol concentration in the control group in measurement 3 subjects and increased by $10 \%$ in 7 subjects (Fig. 8). In the study group of rabbits, the average blood glucose concentration was $83-143 \mathrm{mg} / \mathrm{dl}$ in Stage I, 94-140 $\mathrm{mg} / \mathrm{dl}$ during tonic immobility and $93-131 \mathrm{mg} / \mathrm{dl}$ at the end of the clinical trial (Fig. 7). In analysing the results obtained in Stages I and III, it was found that glucose concentration decreased during the observation period by an average of $7 \%$ in 7 rabbits of the study group and increased by an average of $16 \%$ in 3 rabbits (Fig. 8).

Blood serum cortisol concentration. The concentration of cortisol in the blood serum of the control group animals was $3.6 \mu \mathrm{g} / \mathrm{dl}$ in Stage I and 1.51-7.62 $\mu \mathrm{g} / \mathrm{dl}$

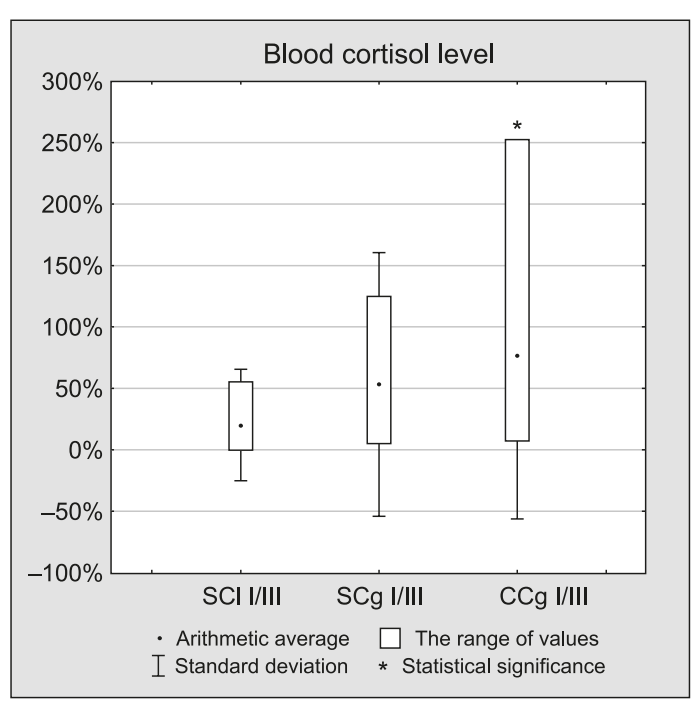

Fig. 10. Percentage change in cortisol concentration between initial measurement and measurement during tonic immobility - I/II; between measurement during tonic immobility and final measurement - II/III; between initial measurement and final measurement - I/III Explanations: $\mathrm{S}$ - study group; $\mathrm{C}$ - control group; 1 -decrease in value; $\mathrm{g}$ - increase in value. 
in Stage III, at the end of the observation (Fig. 9). In all rabbits of this group, cortisol concentration in blood increased by an average of $78 \%$ over the observation period (Fig. 10). The mean cortisol concentration in the rabbits of the study group was 1-2.9 $\mu \mathrm{g} / \mathrm{dl}$ in Stage I and $1-359 \mu \mathrm{g} / \mathrm{dl}$ in Stage III, at the end of the clinical trial (Fig. 9). In the study group, cortisol concentration decreased by an average of 20\% in 6 rabbits and increased by an average of 53\% in 4 rabbits (Fig. 10).

Rabbits are becoming increasingly frequent patients in veterinary clinics, where stress induction is relatively easy. Staff in veterinary clinics and practices specialising in rabbit treatment should have knowledge of the behaviour of these animals, and all the procedures to which they are subjected should be as short as possible and kept to the necessary minimum.

So far, except for one study by McBride, there have been no observations of stress caused by TI. Therefore, the harmfulness of the TI procedure for these animals has not been proven (18).

It has been repeatedly stated that the heart rate and heart rate measurements in rabbits are related to the way in which tachycardia or bradycardia may be recorded in these animals $(9,10)$. The development of bradycardia, together with reduced respiratory rate and significant pupil dilation, indicates stimulation of the sympathetic nervous system during TI (13).

The main stress marker, serum cortisol concentration, increased in all control group rabbits and only in 4 study group animals. This indicates that corticoliberin $(\mathrm{CRH})$ and adrenocorticotropin (ACTH) are not produced during TI, which means that lying on the back is not a stress factor for most rabbits. A study by Giannico et al. (11) showed that rabbits with a more active adrenal cortex and higher serum cortisol concentration collapse in TI more easily, which was the basis for the conclusion that tonic immobility is an antagonistic reaction to stress (9).

Similarly, the glucose concentration in the control group increased between the first and third measurements, whereas a general decrease in serum concentration was observed in the control group rabbits. In rabbits, as in other animal species, hyperglycaemia can be caused by stress. In a study by Harcourt-Brown, 58\% of rabbits showing signs of hyperglycaemia also had elevated levels of this sugar (12). Although there are no reports of the exact mechanism by which hyperglycaemia develops, many authors confirm its occurrence in animals exposed to pain or situations that may cause stress, such as transport and some research procedures $(12,17)$.

Our observations allowed us to conclude that those of the rabbits admitted to the veterinary clinic which underwent clinical examination in a standard position were more stressed than the animals under TI. Tonic immobility induced by inversion of the animal on its back is a safe method of rabbit restraint, greatly facilitating clinical examination and other diagnostic procedures for veterinarians and minimising the risk of injury due to improper taming methods.

\section{References}

1. Alboni M., Bertorelle G.: The origin of vasovagal syncope: to protect the heart or to escape predation? Clin. Auton. Res. 2008, 18, 170-178, doi:10.1007/ s10286-008-0479-7.

2.Bivin W. S.: Basic biomethodology, [in:] Manning P. J., Ringler D. H., Newcomer C. E. (eds.): The Biology of the Laboratory Rabbit, $2^{\text {nd }}$ edn. Academic Press. 1994, p. 72-86, doi: 10.1016/C2013-0-11681-9.

3. Carrive P.: Dual activation of cardiac sympathetic and parasympathetic components during conditioned fear to context in the rat. Clin. Exp. Pharmacol. Physiol. 2006, 33, 1251-1254, doi: 10.1111/j.1440-1681.2006.04519.x.

4. Chertok L.: Animal hypnosis, [in:] Fox M. W. (ed.): Abnormal behavior in animals. Saunders W. B., Philadelphia 1968, 129-158.

5. Coutinho R., Da Silva L. F., Menescal-de-Oliveira L.: Modulation of tonic immobility in guinea pig PAG by homocysteic acid, a glutamate agonist. Physiol Behav. 2008, 94, No. 3, 468-473, doi: 10.1016/j.physbeh.2008.02.013.

6. Da Silva L. F., Menescal-de-Oliveira L.: Role of opioidergic and GABAergic neurotransmission of the nucleus raphe in guinea pigs. Brain Res. Bull. 2007, 72, No. 1, 25-31, doi: 10.1016/j.brainresbull.2006.12.005.

7. Fanselow M. S.: Neural organisation of defensive behaviour system responsible for fear. Psychon. Bull Rev. 1994, 1, 429-438, doi: 10.3758/BF03210947.

8. Gallup G. G. Jr: Genetic influence on tonic immobility in chickens. Ann. Learn. Behav. 1974, 2, 145-147, doi: 10.3758/BF03199142.

9. Giannico A. T., Lima L., Lange R. R., Froes T. R., Montiani-Ferreira F.: Proven cardiac changes during death-feigning (tonic immobility) in rabbits (Oryctolagus cuniculus). J. Comp. Physiol. A Neuroethol. Sens Neural. Behav. Physiol. 2014, 200, 305-310, doi: 10.1007/s00359-014-0884-4.

10. Giannico C.: Blood pressure and heart rate in the rabbit during animal hypnosis. Electroencephalogr. Clin. Neurophysiol. 1974, 37, 231-237, doi: 10.1016/0013-4694(74)90026-1.

11. Giannico C., Farbollini F., Lupo di prisco C.: Plasma corticosterone and its relation to susceptibility to animal hypnosis in rabbits. Neurosci. Lett. 1979, 11, 971-274, doi: 10.1016/0304-3940(79)90006-5.

12. Harcourt-Brown F. M., Harcourt-Brown S. F. Clinical value of blood glucose measurement in pet rabbits. Vet. Rec. 2012, Jun 30; 170 (26), 674, doi: 10.1136/ vr.100321.

13. Hatton D. C., Woodruff M., Meyer M. E.: Cholinergic modulation of tonic immobility in the rabbit (Oryctolagus cuniculus). J. Comp. Physiol. Psychol. 1975, 89, 1052-1060, doi: 10.1037/h0077191.

14. Henningsen A. D.: Tonic immobility in 12 elasmobranchs: use as an aid in captive husbandry. Zoo. Biol. 1994, 13, 325-332, doi: 10.1002/zoo.1430130406.

15. Klemm W. R.: Behavioral arrest: in search of the neural control system. Progr. Neurobiol. 2001, 65, 453-47, doi: 10.1016/S0301-0082(01)00016-8.

16. Marx B. P., Forsyth J. P., Gallup G. G., Fusé T., Lexington J. M.: Tonic immobility as an evolved predator defense: implications for sexual assault survivors. Clin. Psychol. Sci. Pract. 2008, 15, 74-90, doi: 10.1111/ j.1468-2850.2008.00112.x.

17. Mayer J., Schnellbacher R., Rich E., Divers S., Ward C.: Use of a Commercial Continuous Interstitial Glucose Monitor in Rabbits (Oryctolagus Cuniculus). J. Exot. Pet. Med. 2016, 25, 3, 220-225, doi: 10.1053/j.jepm.2016.04.006.

18. McBride A., Day S., McAdie T., Meredith A., Barley J., Hickman J., Lawes L.: Trancing Rabbits: relaxed hypnosis or a state of fear? Conference: VDWE International Congress on Companion Animal Behaviour and Welfare January 2006.

19. Overeem S., Lammers G. J., Dijk J. G.: Cataplexy: 'tonic immobility' rather than 'REM-sleep atonia? Sleep Med. 2002, 3, 471-477, doi: 10.1016/ S1389-9457(02)00037-0.

20. Ratner S. C.: Comparative aspects of hypnosis, [in:] Gordon J. E. (ed.) Handbook of clinical experimental hypnosis. New York: Macmillan 1967.

21. Roshchina G. Ya., Email V. I., Koroleva V. I., Davydov V I.: Changes in the High-Frequency Activity of Rabbit Brain Biopotentials in the State of "Animal Hypnosis" 2011, October 41, 772, doi: 10.1007/s11055-011-9486-6.

22. Rusinova V., Davydov V. I.: Dynamics of changes in electrical activity in the rabbit cerebral cortex during sequential sessions of "animal hypnosis". Neurosci. Behav. Physiol. 2010, 40, 471-478, doi: 10.1007/s11055-010-9283-7.

23. Rusli $U$., $W u$ N., Booth D.: Tonic Immobility in Newly Emerged Sea Turtle Hatchlings Chelonian Conservation and Biology 2016, 15 (1), 143-147, doi: 10.2744/CCB-1185.1.

24. Sanberg P., Ossenkopp K. P., Kavaliers M.: Motor Activity and Movement Disorders Research Issues and Applications. Springer Science Business Media, LLC Humana Press 1996, 57-60.

25. Watsky M. A., Gruber S. H.: Induction and duration of tonic immobility in the lemon shark. Negaprion brevirostris. Fish Physiol. Biochem. 1990, 8, 207-210, doi: 10.1007/BF00004459.

Corresponding author: Anna Wilczyńska DVM, Department of Epizootiology and Clinic of Infectious Diseases, Faculty of Veterinary Medicine, University of Life Sciences in Lublin, ul. Głęboka 30, 20-612 Lublin, Poland; e-mail: ania.osinska@eyosys.pl 